\title{
A global view of livestock biodiversity and conservation - GLOBALDIV
}

\author{
P. Ajmone-Marsan* and The GLOBALDIV Consortium ${ }^{+}$ \\ ${ }^{*}$ Institute of Zootechnics, Università Cattolica del Sacro Cuore, via Emilia Parmense 84, 29122 Piacenza, Italy. ${ }^{\dagger}$ http://www.globaldiv.eu
}

\section{Summary}

\begin{abstract}
GLOBALDIV - A global view of livestock biodiversity and conservation - is a three- year project funded by the European Commission in the framework of the AGRI GEN RES initiative. It is formed by a core group of partners who participated in past EU or continental scale projects on Farm Animal Genetic Resources characterization and conservation. It also involves a much larger number of experts that are actively contributing to the success of the iniziative. The project aims at improving the conservation, characterisation, collection and utilisation of genetic resources in agriculture in EU and beyond, complementing and promoting work undertaken in the Member States at the Community level and facilitating co-ordination of international undertakings on genetic resources in agriculture.
\end{abstract}

Keywords biodiversity, conservation, livestock.

\section{Introduction}

Biodiversity forms the basis of life on earth. The Convention on Biological Diversity, adopted at the United Nations Conference on Environment and Development held in Rio de Janeiro in 1992, states that the contracting Parties are 'conscious of the intrinsic value of biological diversity' and 'conscious also of the importance of biological diversity for evolution and for maintaining life sustaining systems of the biosphere.' The Parties affirm that 'the conservation of biological diversity is a common concern of humankind' and that they are 'aware that conservation and sustainable use of biological diversity is of critical importance for meeting the food, health and other needs of the growing world population'.

For about 10000 years, farmers have been managing their livestock in different environments, leading to animals that are well adapted to local conditions. About 200 years ago, the concept of breeds emerged. Within a breed, animals were selected for uniform visible characteristics and most crossings were of animals with similar phenotypes or genetic background. Over the past few decades, selection pressure was again increased, to increase production via the application of quantitative genetics methods, with little

Address for correspondence

P. Ajmone-Marsan, Institute of Zootechnics, Università Cattolica del Sacro Cuore, Piacenza, Italy.

E-mail: paolo.ajmone@unicatt.it

Accepted for publication 17 November 2009 regard for the preservation of genetic diversity. Several breeds now suffer from the consequences of inbreeding, with effective population sizes sometimes falling below 50. In addition, the most productive breeds are becoming more and more dependent on intensive management, while farmers are abandoning the more robust traditional breeds. As a consequence, genetic resources of livestock are threatened by erosion. The situation is most alarming for developing countries, where industrial breeds often replace local breeds that have unique disease resistance and adaptations to the local environment.

In September 2007 at Interlaken, Switzerland, country delegates from all over the world adopted a global plan of action for conserving indigenous farm animal genetic resources, FAnGR (FAO 2007). At this meeting, the notion emerged that livestock diversity is decreasing at an accelerated pace, with many breeds being lost throughout the world. Furthermore, populations are poorly characterized in developing countries, where local livestock diversity represents a unique resource for productivity and local breeding programmes and also provides a major pathway out of poverty.

Conservation of genetic diversity is now universally accepted as being vital for sustainable management of these resources. It can be accomplished by in situ preservation of endangered and valuable breeds, selection programmes that will restore genetic diversity in industrial breeds, or the cryo-conservation of gametes, embryos and somatic cells of the existing gene pool.

Scientific research into genetic diversity followed closely the rapid advances in molecular genetics. However, as 
sustainable conservation implies strategies for the valuation of FAnGR in their environment, it needs the convergence of different scientific disciplines, spanning genetics through socio-economics to GIScience.

It is also essential that the need to manage genetic diversity in livestock populations is brought to the attention of the general public, policy makers and, last but not least, the new generation of scientists. This calls for an effective dissemination of new technologies for measuring phenotypic traits, low-cost genomic analysis and new statistical and computational tools.

\section{The GLOBALDIV project}

GLOBALDIV - A global view of livestock biodiversity and conservation - is a 3-year project funded by the European Commission in the framework of the AGRI GEN RES initiative. It is formed by a core group of partners who participated in past EU or continental-scale projects on FAnGR characterization and conservation. It also involves a much larger number of experts who are actively contributing to the success of the initiative (Table 1). Among them are representatives of the European Association for Animal Production (EAAP), the Food and Agriculture Organization of the United Nations (FAO), the International Livestock Research Institute (ILRI) and the International Agency of Atomic Energy (IAEA). The IAEA presently coordinates several projects on livestock diversity in developing countries. Specific objectives of the GLOBALDIV project are: (i) the integration and dissemination of experience gained in past large-scale biodiversity projects; (ii) a review of methods to organize and combine existing data and databases to obtain a global (worldwide) view of diversity of livestock species; (iii) the promotion of the formation of interdisciplinary teams of experts reviewing key aspects of Animal Genetic Resource characterization,

Table 1 Members of the GLOBALDIV Consortium.

\begin{tabular}{|c|c|c|c|}
\hline Name & Affiliation & Country & Role \\
\hline P. Ajmone-Marsan & Università Cattolica del Sacro Cuore, Piacenza & IT & Coordinator \\
\hline J.F. Garcia & Universidade Estadual Paulista, Aracatuba & BR & Partner \\
\hline J. Han & International Livestock Institute and Chinese Academy of Science, Beijing & INTL, CN & Partner \\
\hline O. Hanotte & International Livestock Institute, Nairobi and Nottingham University & INTL, UK & Partner \\
\hline S. Joost & LASIG/EPFL and MicroGIS, Lausanne & $\mathrm{CH}$ & Partner \\
\hline J.A. Lenstra & University of Utrecht, Utrecht & $\mathrm{NL}$ & Partner \\
\hline A. Rosati & European Association for Animal Production, Rome & INTL & Partner \\
\hline S. Weigend & Friedrich-Loeffler-Institut, Neustadt & $\mathrm{DE}$ & Partner \\
\hline P. Baret & Faculté D’Ingénierie Biologique, Agronomique et Environnementale, Louvain-la-Neuve & $\mathrm{BE}$ & Expert \\
\hline B. Besbes & Food and Agriculture Organization of the United Nations, Rome & INTL & Expert \\
\hline P. Boettcher & Food and Agriculture Organization of the United Nations, Rome & INTL & Expert \\
\hline M. Bruford & Cardiff University, Cardiff & UK & Expert \\
\hline L. Colli & Università Cattolica del Sacro Cuore, Piacenza & $\mathrm{IT}$ & Expert \\
\hline S. Dunner & Madrid University, Madrid & ES & Expert \\
\hline H. Eding & Animal Evaluations Unit, CRV, Arnhem & $\mathrm{NL}$ & Expert \\
\hline G. Gandini & Milano University, Milano & IT & Expert \\
\hline E. Groeneveld & Friedrich-Loeffler-Institut, Neustadt & DE & Expert \\
\hline L.F. Groeneveld & Friedrich-Loeffler-Institut, Neustadt & DE & Expert \\
\hline G. Hewitt & University of East Anglia, Norwich & UK & Expert \\
\hline S. Hiemstra & Wageningen University, Wageningen & $\mathrm{NL}$ & Expert \\
\hline I. Hoffmann & Food and Agriculture Organization of the United Nations, Rome & INTL & Expert \\
\hline J. Kantanen & MTT Agrifood Research, Jokioinen & $\mathrm{FI}$ & Expert \\
\hline M. Malek & International Agency of Atomic Energy (IAEA), Wien & INTL & Expert \\
\hline R. Negrini & Università Cattolica del Sacro Cuore, Piacenza & $\mathrm{IT}$ & Expert \\
\hline M. Pellecchia & Università Cattolica del Sacro Cuore, Piacenza & IT & Expert \\
\hline J. Roosen & Munich Technical University, Munich & $\mathrm{DE}$ & Expert \\
\hline H. Simianer & Georg August University, Göttingen & $\mathrm{DE}$ & Expert \\
\hline P. Taberlet & Université J. Fourier and CNRS, Grenoble & FR & Expert \\
\hline H. Täubert & Georg Austin University, Göttingen & DE & Expert \\
\hline M. Tixier-Boichard & Istitut National de la Recerche Agronomique (INRA), Jouy en Josas & FR & Expert \\
\hline M. Toro & Universidad Politécnia, Madrid & ES & Expert \\
\hline A. Valentini & Tuscia University, Viterbo & IT & Expert \\
\hline J.L. Williams & Parco Tecnologico Padano, Lodi & $\mathrm{IT}$ & Expert \\
\hline M. Zjalic & European Association for Animal Production, Rome & INTL & Expert \\
\hline
\end{tabular}


evaluation and conservation; (iv) a review of methods and software for integration of specialist knowledge from disciplines as different as husbandry, genetics, sociology, economics and geographic analysis; (v) the identification of future research opportunities and priorities for the characterization, evaluation and sustainable conservation of livestock species; (vi) the training of technical and scientific personnel involved at various levels in FAnGR characterization and conservation; and (vii) a wide dissemination of results to all relevant stakeholders in the Livestock Farming Sector.

To reach these objectives, partners and experts are organized in Working Groups (WGs) addressing specific scientific aspects relevant to conservation.

\section{Characterization of FAnGR diversity}

A thorough investigation of genetic variation in a given species would provide valuable information for conservation purposes. Breeds need to be characterized for phenotypic traits and variables associated with their production environment. These are indicators of the functional genetic diversity carried by an individual or population. Diversity at the molecular level can be detected via variable DNA markers.

Studies of mitochondrial DNA, microsatellite DNA profiling and Y-chromosomes have revealed many details about the process of domestication, the diversity retained by breeds and the relationships between breeds. However, so far, we have only a small part of the genetic information and many essential questions remain unanswered. Therefore, the advent of new technologies is most timely. High-throughput SNP (single nucleotide polymorphism) genotyping is now rapidly gaining ground and will give a more detailed picture of the genetic diversity. Meanwhile, we are witnessing the next revolution in genetics: the development of techniques for the affordable sequencing of complete genomes, for which several alternative technologies have already passed the proof-of-principle stage.

The Working Group addresses topics related to the characterization of FAnGR with the aim of setting operational standards for data collection, molecular tools and data analysis methods. In addition, it delivers information that is most relevant for the revision of the current FAO reference guidelines for conservation.

\section{Meta-analysis of diversity}

Combined analysis of microsatellite data obtained in separate studies would be highly desirable. Most population genetic studies are limited to a restricted small number of breeds, often from a single country (Baumung et al. 2004). In addition, different sets or different subsets of the FAO recommended markers have often been used, while for the common markers no standard samples are typed across projects to correct for variation in allele size assignments. To promote the use of common markers, FAO is now proposing an updated ranked list of microsatellite loci in major livestock species (http://dad.fao.org/cgi-bin/getblob.cgi?sid $=01 \mathrm{e} 98 \mathrm{e} 40743 \mathrm{a} 456 \mathrm{~d} 0 \mathrm{e} 3477592 \mathrm{f} 124 \mathrm{be} 6,50005882$ [last accessed on 24 March 2010]). FAO recommends the use of these markers in order of ranking to maximize the number of common markers in independent investigations.

Ongoing close coordination between previous and current large scale projects promises the delivery of a global estimate of diversity in the near future. This requires new methods of meta-analysis to combine datasets that have only a few markers and breeds in common (Freeman et al. 2006). The resulting global perspective of livestock diversity will be extremely valuable for a reconstruction of the origin and history of domestic animal populations and indirectly of human populations. Also, it will highlight regional and local hotspots of diversity where conservation efforts will be most effective. Last, but not least, it will allow an assessment of the diversity of European livestock in a global context.

The Working Group aims to define recommendations, methods and limits in the joining of independent datasets either having common sets of markers/populations or being completely independent. In addition, it formulates proposals for arriving at an international standardization of a SNP panel for future diversity studies. Ongoing implementations of the most advanced genetic markers, SNPs and CNVs (copy number variation) have been designed for mapping production traits, and, by including markers identified in the Western cosmopolitan breeds, are probably less suitable for characterization of diversity in local breeds.

\section{Socio-economic aspects of conservation}

The importance of establishing the value of genetic resources is explicit in the first article of the International Convention on Biological Diversity (CBD 1992) that states that 'The objectives of this Convention, to be pursued in accordance with its relevant provisions, are the conservation of biological diversity, the sustainable use of its components and the fair and equitable sharing of the benefits arising out of the utilization of genetic resources, including by appropriate access to genetic resources and by appropriate transfer of relevant technologies, taking into account all rights over those resources and to technologies, and by appropriate funding'.

Thus, the economic valuation of FAnGR potentially plays a key role in developing effective breeding programmes or conservation programmes and finally in the development of policy and management decisions.

Under intensive animal breeding, the economic value of genetic improvement of most species lies in productivity, plus a small value for survival and reproduction characteristics and occasionally also a small component in disease 
resistance and product quality. Conversely, in low input systems, the highest value lies in adaptation, disease resistance and in other functions such as traction and nutrient recycling. This means that low input production systems typically require a greater range of genetic attributes. Hence, the value of livestock breeds is not captured completely by the market value. For this reason, methods for evaluating livestock biodiversity have to go beyond the marketplace and consider the changing agricultural practices, the intensification of production systems, the use of higher producing but less well-adapted breeds, crossbreeding and genetic erosion, as well as national and international policy (including de-coupling).

The Convention on Biological Diversity stated that conservation should be ideally in situ, with ex situ conservation being less desirable and in vitro conservation being used only in emergency situations (FAO, 1998). However, reviews of $e x$ situ, in situ and in vitro conservation programmes and assessment of their relative efficiency and costs for gametes and embryos, including cost of re-establishment of the original population, may be of interest, and these conclude that the future use of in vitro methods as an effective conservation strategy to back-up maintenance of live animals.

This Working Group addresses specific topics related to the economic aspects of the conservation of FAnGR, such as the evaluation of efficiency and economic aspects of in situ and $e x$ situ conservation, the identification of case studies of sustainable conservation that are relevant to other countries, and a critical analysis of current CAP (Common Agricultural Policy) and structural fund programmes.

\section{Integrating data and setting priorities for conservation}

The application of molecular technologies to the issues of genetic diversity and conservation is producing a huge amount of genetic information. New statistical approaches are being developed to exploit and finally combine such enlarged datasets. Although this information represents the basis of any conservation study, a complete picture requires the integration of data from different disciplines, such as climatology, geography, sociology and economic sciences. Such an integrated approach will lead to the definition of appropriate guidelines for the management and conservation of genetic resources.

The first step of the integration process is linking the genetic information to landscape variables. This increases the power of the genetic data by exploiting the spatial dimension of the information provided by GIS technologies. In fact, GIScience offers multiple ways for analysing spatial information, each of them being closely linked to a geographic database.

Despite the increasing interest in and the importance attributed to FAnGR, research has provided little guidance as to how conservation programmes should select valuable breeds and populations. Weitzman (1992, 1993, 1998) recognized that biodiversity conservation is an inherently economic question and provides a framework for decisions. While his framework is of high generality and is applicable to many diversity conservation questions, he was primarily interested in the conservation of species diversity. However, FAnGR conservation is primarily at the level of breed or population. The application of the Weitzman model to the prioritization of breeds for conservation has been challenged in recent years. First, diversity measures based on betweenbreed distances ignore the within-breed diversity, i.e. the genetic variation within a breed. Within-breed diversity contributes considerably to the total diversity within a species and is most important as it influences the reproductive viability of a breed and its adaptability to changing environmental conditions. In fact, within-breed diversity at neutral loci often accounts for $70 \%$ or more of total genetic variance. Secondly, genetic drift in breeds with small population sizes creates large genetic distances from other breeds, which then leads to an exaggeration of their contribution to the diversity via the Weitzman model.

Thirdly, with one exception (Simianer 2002), none of the applications considers other dimensions of human welfare stemming from the conservation of breeds. This is a major drawback, as society is not blindly conserving diversity for its own sake. In fact, political decisions on conservation measures are made under the constraints of the budget allocated to these programmes. This implies that the question of how to optimally allocate the available funding is most relevant. Available information on the specific merits of different livestock breeds in varied or highly specialized production systems, their genetic and phenotypic characteristics, socio-economic value and local context will all be relevant for conservation measures.

This Working Group delivers information on the tools available for integrated analysis and prioritization, and recommendations for their use as decision aids. Decision aids help decision makers to understand the consequences of alternative courses of action and to decide upon optimal allocation of finite resources available for conservation, but cannot substitute for human decisions.

\section{Future needs and perspectives}

All Working Groups are about to combine their knowledge and experience to fulfil this final task of GLOBALDIV, with three main objectives: (i) to identify gaps in knowledge, methods and tools available for the overall characterization, valuation and conservation of FAnGR; (ii) to review the perspectives of innovative methods, including -omics technologies, nanotechnologies, genetic modification, information from genomic projects, etc. in addressing needs for characterization and conservation activities and in offering new opportunities for the genetic improvement of FAnGR; and (iii) to define an agenda for future research activities, listing topics to be studied in further detail in different areas. 


\section{Dissemination}

The outcome of the brainstorming and expertise of the Working Groups is disseminated towards different stakeholders through the GLOBALDIV website (http://www. globaldiv.eu), a newsletter (to date 13 issues have been disseminated through the web), the EAAP and DAD-IS mailing lists, workshops (the first workshop was held in Bydgoszcz, in March 2009, and a second is planned in Rome, 5-6 May 2010), summer schools (two programmes have been organized in Piacenza in September 2008 and 2009 and were attended by students hailing from more than 30 countries of all five continents), and review papers, which aim to disseminate updated state of the art information on aspects relevant to FAnGR characterization and conservation throughout the scientific community.

\section{Animal Genetics special issue}

This special issue includes four of the review papers delivered so far by the GLOBALDIV WGs.

The first paper by Linn Groeneveld et al. reviews the present status of FAnGR characterization in many species (cattle, water buffalo, goats, sheep, horses, pigs, camelids, yak, and chickens) and describes databases hosting data on breed description, including demographic, environmental, phenotypic and genetic information. The paper also discusses the present and future needs in terms of infrastructure for a comprehensive analysis of genetic diversity in livestock species.

The second paper by Irene Hoffman gives an overview of the mutual influence of livestock breeding and climate change. Livestock influence the climate by contributing to pollution and greenhouse gas emission. Climate changes affect live-stock by decreasing water and pasture availability and increasing global temperature and the risk of disease. This paper discusses global trends, with a particular focus on developing countries and on possible measures that can be adopted to mitigate the adverse effects.

The third paper from Stephane Joost et al. discusses problems linked to the integration of data from different disciplines for the description and management of FAnGR. The paper describes the potential of GIScience for data integration, with examples of successful GIS application in description and integration of multidimensional data.

In the last paper, Paul Boettcher et al. critically review methods for setting priorities in FAnGR conservation. The paper describes different approaches and discusses the key issue of the importance of within- vs. between-breed diversity in setting conservation priorities, which is presently the main criticism of the Weitzman approach. A final table summarizes the main features of the most popular freeshare software for the analysis of genetic diversity.

\section{Conclusion}

We have to face the fact that FAnGR extinction and threats will probably continue at a worrying pace. The scientific community cannot let this trend continue unnoticed nor ignore its consequences. We hope that an increasing number of scientists will be interested in FAnGR characterization and conservation issues. We trust their efforts to inform decision makers and the general public on the most undesirable loss of diversity and to share strategies for the conservation of FAnGR for future generations.

\section{Acknowledgements}

The Author states that there is no conflict of interest regarding the material discussed in the manuscript. Action GLOBALDIV AGRI GEN RES 067 receives financial support from the European Commission, Directorate-General for Agriculture and Rural Development, under Council Regulation (EC) No 870/2004.

\section{References}

Baumung R., Simianer H. \& Hoffmann I. (2004) Genetic diversity studies in farm animals - a survey. Journal of Animal Breeding and Genetics 121, 361-73.

Convention on Biological Diversity (1992) United Nation Treaty Series N. 30619. Available at: http://www.cbd.int/convention/ convention.shtml, last accessed on 24 March 2010.

FAO (1998) Secondary Guidelines for Development of National Farm Animal Genetic Resources Management Plans. Management of Small populations at Risk. FAO, Rome, Italy. Available at: http:// www.fao.org/docrep/010/a1250e/a1250e00.htm, last accessed 24 March 2010.

FAO (2007) The State of the World's Animal Genetic Resources for Food and Agriculture. FAO, Rome, Italy. Available at: http:// www.fao.org/docrep/010/a1250e/a1250e00.htm, last accessed 24 March 2010.

Freeman A.R., Bradley D.G., Nagda S., Gibson J.P. \& Hanotte O. (2006) Combination of multiple microsatellite data sets to investigate genetic diversity and admixture of domestic cattle. Animal Genetics 37, 1-19.

Simianer H. (2002) Noah's dilemma: which breeds to take aboard the ark? 8th World Congress on Genetics Applied to Livestock Production 26, 2 .

Weitzman M.L. (1992) On diversity. Quarterly Journal of Economics 107, 363-405.

Weitzman M.L. (1993) What to preserve? An application of diversity theory to crane conservation. Quarterly Journal of Economics 108, 157-83.

Weitzman M.L. (1998) The Noah's ark problem. Econometrica 66, 1279-98. 